\title{
FIELDS AND ELEMENTARY PARTICLES
}

\section{EXPANSION OF THE TWO-PARTICLE DIRAC EQUATION IN POWERS OF $1 / c$ TO HIGHER ORDERS}

\section{A.I. TUROVSKY}

PACS 02.30.Mv, 03.65.Pm (C) 2011

\author{
Bogolyubov Institute for Theoretical Physics, Nat. Acad. of Sci. of Ukraine
}

(14b, Metrolohichna Str., Kyiv 03680, Ukraine; e-mail: turovsky@bitp. kiev.ua)

Using an extension of the Foldy-Wouthuysen transformation to two-particle wave equations, we consider the problem of expansion of the two-body Dirac Hamiltonian in powers of $1 / c$ up to the fourth order. The transformed Hamiltonian in an even-even form to order $1 / c^{4}$ is obtained. It is shown that the extra terms which can be eliminated by additional unitary transformations appear in the expansion in higher orders. As an example, the Breit equation for Coulomb particles is considered, and all the terms of order $1 / c^{4}$ in its reduction are calculated. The obtained results can be used for the expansion of relativistic and quasirelativistic two-particle wave equations to higher orders.

\section{Introduction}

Nowadays, the relativistic and quasirelativistic twoparticle wave equations are widely used in a study of various relativistic effects in quantum systems and in calculations of their energy spectra [1]. In many cases, however, to obtain an acceptable description of a twobody system, it is not essential to solve the original equations of motion, and one can often restrict oneself to the consideration of their approximate forms. In order to reduce the relativistic equations to such forms, canonical transformations of the Foldy-Wouthuysen (FW) type are usually applied. At first, the FW transformation was developed to expand relativistic one-particle equations of the Dirac type into a series in powers of $1 / c$ (or $1 / \mathrm{m}$ ) [2-4], and it was generalized afterwards by Chraplyvy to two-particle equations of the Breit type [5-8]. This method allows one to expand a relativistic Hamiltonian to any desired degree of approximation, keeping its Hermitian character.

As a rule, it is sufficient for many applications to limit oneself to the expansion of two-particle wave equations in powers of $1 / c$ to the second order. Thus, the well- known Breit-Fermi Hamiltonian which can be derived from the nonrelativistic reduction of equations of the Breit or the Bethe-Salpeter type is often used to describe spectra of atomic and quark systems [7,9]. Yet, a further expansion of relativistic and quasirelativistic two-body equations to higher orders in $1 / c$ can be of some interest as well, and not only from the theoretical point of view. The improvements of experimental techniques for the study of the energy spectra in atomic systems, first of all, in hydrogen-like atoms, muonium, and positronium, enable one to make precise measurements of their energy levels [10]. Researchers usually apply quantum electrodynamics (QED) to calculate the contributions of orders $\alpha^{5} m c^{2}$ and $\alpha^{6} m c^{2}$ to the energy (see, e.g., [11] and references therein). However, the problem of derivation of the higher-order Hamiltonian for an arbitrary light atom within the framework of QED still remains a difficult one. Nevertheless, the direct derivation of an effective Hamiltonian that contributes to $\alpha^{6} m c^{2}$ can be performed by the reduction of relativistic two-body equations, though it does not give a complete treatment of the field effects.

In this paper, we consider the problem of expansion of the two-particle Dirac equation to order $1 / c^{4}$ by applying an extension of the FW method to two-body systems for the case of unequal masses. In Section 2, a short review of the $\mathrm{FW}$ transformation and its generalization to the two-body problem is given. Section 3 deals with the higher-order transformed Hamiltonian, for which all the $1 / c^{4}$-order terms are found. It happens that this Hamiltonian involves certain terms having mass differences in the denominators even if both particles are in a positive or negative energy state. We propose the additional unitary transformations canceling these extra terms. In Section 4 , we calculate all the terms of order $1 / c^{4}$ in the 
expansion of the Breit equation for Coulomb particles. The problem of modification of the fourth-order part of the transformed Hamiltonian is also discussed. Finally, Section 5 contains main conclusions of the work.

\section{Summary of the FW Transformation and Its Extension to Two-Particle Systems}

We start off with a brief review of the FW method which is the nonrelativistic expansion of the Dirac Hamiltonian for a particle in external fields [2-4]. One can express this Hamiltonian in terms of even $\mathcal{E}$ and odd $\mathcal{O}$ four-by-four matrices. An effect of even matrices upon components of a four-component spinor produces a new spinor, in which the upper and lower components of the first one are not interlinked with one another. An effect of odd matrices upon a spinor interlinks its upper and lower components. The identity and $\beta$ matrices are even, and $\alpha_{x}, \alpha_{y}, \alpha_{z}$ are odd. Thus, the Dirac Hamiltonian for a single particle in external scalar $V$ and vector $\left(B_{0}, \mathbf{B}\right)$ fields can be written down as follows:

$H=\beta m c^{2}+\mathcal{E}+\mathcal{O}$.

Here, $\mathcal{E}=B_{0}-\beta V$ is the even part, $\mathcal{O}=\boldsymbol{\alpha}(c \mathbf{p}-\mathbf{B})$ is the odd one, and $\beta \mathcal{E}=\mathcal{E} \beta, \beta \mathcal{O}=-\mathcal{O} \beta$. In order to remove all the odd terms, the original Hamiltonian (1) must be subjected to canonical transformations of the type

$e^{i S} H e^{-i S}=H+i[S, H]+\frac{(i)^{2}}{2 !}[S,[S, H]]+\ldots$

$\ldots+\frac{(i)^{n}}{n !}[S,[S, \ldots,[S, H] \ldots]]+\ldots$,

where the generating function $S$ is suitably chosen. Here, the reciprocal speed of light or the reciprocal mass may be taken as the expansion parameter of the series.

Though one cannot find a unitary transformation canceling all of the odd terms in all orders in the case of external fields, the one-particle Dirac Hamiltonian can be converted into an even operator, to any desired degree of approximation, by canonical transformations like (2). To remove the odd terms in $H$, the function $S$ is usually chosen for the first iteration in the form

$S=-\frac{i \beta}{2 m c^{2}} \mathcal{O}$.

By removing the $\mathcal{O}$ terms, the transformation produces new even and odd ones instead, but all of the latter are of lower orders of magnitude. Using (2) and (3), $H$ can be converted into an even operator

$$
\begin{aligned}
& H_{\mathrm{tr}}=\beta m c^{2}+\mathcal{E}+\frac{\beta}{2 m c^{2}} \mathcal{O}^{2}-\frac{1}{8 m^{2} c^{4}}[\mathcal{O},[\mathcal{O}, \mathcal{E}]]- \\
& -\frac{\beta}{8 m^{3} c^{6}} \mathcal{O}^{4}-\frac{\beta}{8 m^{3} c^{6}}[\mathcal{O}, \mathcal{E}]^{2}+\frac{1}{12 m^{4} c^{8}}\left[\mathcal{O}^{3},[\mathcal{O}, \mathcal{E}]\right]+ \\
& +\frac{1}{384 m^{4} c^{8}}[\mathcal{O},[\mathcal{O},[\mathcal{O},[\mathcal{O}, \mathcal{E}]]]]+\frac{\beta}{16 m^{5} c^{10}} \mathcal{O}^{6}+\ldots,
\end{aligned}
$$

where we have retained terms up to order $1 / c^{4}$. In order to get this expansion, one has to do three iterations. We note that there is one term, in which $\mathcal{E}$ is involved twice in the expression (up to order $1 / c^{4}$ ), and it is the only term nonlinear in the scalar interaction. We should also note that the FW transformation is a relatively simple way to get an approximate form of the Hamiltonian to higher orders, and, furthermore, this procedure yields the transformed Hamiltonian in its Hermitian form.

Let us consider an extension of the FW transformation to two-particle wave equations. Following Chraplyvy, we represent the original relativistic Hamiltonian as

$H=\beta_{1} m_{1} c^{2}+\beta_{2} m_{2} c^{2}+(\mathcal{E E})+(\mathcal{E O})+(\mathcal{O E})+(\mathcal{O O})$

It is a sum of the two "large" terms $\beta_{1} m_{1} c^{2}+\beta_{2} m_{2} c^{2}$ and the others which are written down as even-even, evenodd, odd-even, and odd-odd terms, respectively. In general, they are matrices of $16 \times 16=256$ elements and can be represented as direct products of $4 \times 4$ matrices of each particle. Here, we assume that $(\mathcal{E} \mathcal{E}),(\mathcal{O O})$ are of order $c^{0}$ and $(\mathcal{O E}),(\mathcal{E} \mathcal{O})$ are of order $c^{1}$, which corresponds to the case of the two-particle Dirac equation.

As in the case of a single particle, an approximate expression for a two-body Hamiltonian can be obtained by converting it into an even-even operator, by applying transformation (2), where the operator $S$ can be represented as the sum $S=S_{o e}+S_{e o}+S_{o o}$. The generating functions can be written for the first iteration in the form

$$
\begin{aligned}
& S_{o e}=-\frac{i \beta_{1}}{2 m_{1} c^{2}}(\mathcal{O E}), \quad S_{e o}=-\frac{i \beta_{2}}{2 m_{2} c^{2}}(\mathcal{E} \mathcal{O}), \\
& S_{o o}=-\frac{i\left(\beta_{1} m_{1}-\beta_{2} m_{2}\right)}{2\left(m_{1}^{2}-m_{2}^{2}\right) c^{2}}(\mathcal{O O}),
\end{aligned}
$$

because of the meeting of the two large terms in the first commutator of (2). These functions remove the even-odd, odd-even, and odd-odd components from the original Hamiltonian,

$\left[S_{e o}, \beta_{1} m_{1} c^{2}+\beta_{2} m_{2} c^{2}\right]=i(\mathcal{E O})$, 
$\left[S_{o e}, \beta_{1} m_{1} c^{2}+\beta_{2} m_{2} c^{2}\right]=i(\mathcal{O E})$

$\left[S_{o o}, \beta_{1} m_{1} c^{2}+\beta_{2} m_{2} c^{2}\right]=i \frac{m_{1}^{2}-\beta_{1} \beta_{2} m_{1} m_{2}}{m_{1}^{2}-m_{2}^{2}}(\mathcal{O O})+$

$+i \frac{\beta_{1} \beta_{2} m_{1} m_{2}-m_{2}^{2}}{m_{1}^{2}-m_{2}^{2}}(\mathcal{O O})=i(\mathcal{O O})$.

In fact, expressions (6a) resemble $S$ in (3). Operators (6) enable us to transform a sixteen-component twobody equation with the Hamiltonian in the form (5) to its four-component approximate form for chosen energy states of two particles. The generating functions (6) were first proposed by Chraplyvy in [5] to convert a two-body Hamiltonian into an even-even operator to order $1 / c^{2}$. This operator takes a relatively simple form under condition that $(\mathcal{O E})$ and $(\mathcal{E O})$ commute with each other and reads

$$
\begin{aligned}
& H_{A}=\beta_{1} m_{1} c^{2}+\beta_{2} m_{2} c^{2}+(\mathcal{E E})+\frac{\beta_{1}}{2 m_{1} c^{2}}(\mathcal{O E})^{2}+ \\
& +\frac{\beta_{2}}{2 m_{2} c^{2}}(\mathcal{E O})^{2}-\frac{1}{8 m_{1}^{2} c^{4}}[(\mathcal{O E}),[(\mathcal{O E}),(\mathcal{E} \mathcal{E})]]- \\
& -\frac{1}{8 m_{2}^{2} c^{4}}[(\mathcal{E O}),[(\mathcal{E O}),(\mathcal{E E})]]-\frac{\beta_{1}}{8 m_{1}^{3} c^{6}}(\mathcal{O E})^{4}- \\
& -\frac{\beta_{2}}{8 m_{2}^{3} c^{6}}(\mathcal{E O})^{4}+\frac{\beta_{1} \beta_{2}}{4 m_{1} m_{2} c^{4}}\left[(\mathcal{O E}),[(\mathcal{E O}),(\mathcal{O O})]_{+}\right]_{+}+ \\
& +\frac{\beta_{1} m_{1}-\beta_{2} m_{2}}{2\left(m_{1}^{2}-m_{2}^{2}\right) c^{2}}(\mathcal{O O})^{2}
\end{aligned}
$$

where $[a, b]_{+}=a b+b a$. Notice that this expression is linear in $(\mathcal{E} \mathcal{E})$ and $(\mathcal{O O})$ except the last term.

\section{Higher-Order Transformed Hamiltonian}

Expression (8) is the transformed two-body Hamiltonian to the second order. Proceeding with the procedure of transformation of $H$, we obtain new even-even terms which come after $H_{A}$ and are of a lower order of magnitude than those written in (8). They form the higher-order (with respect to $1 / c$ ) part of the transformed Hamiltonian. We will distinguish between two types of terms in it. The $1 / c^{4}$-order terms of the first type are nonlinear in $(\mathcal{E E})$ and $(\mathcal{O O})$ and can be written as

$$
\begin{aligned}
& H_{B}=\frac{-\beta_{1}}{8 m_{1}^{3} c^{6}}[(\mathcal{O E}),(\mathcal{E} \mathcal{E})]^{2}-\frac{\beta_{2}}{8 m_{2}^{3} c^{6}}[(\mathcal{E O}),(\mathcal{E} \mathcal{E})]^{2}+(9 \mathrm{a}) \\
& +\frac{\beta_{1}}{8 m_{1} m_{2}^{2} c^{6}}[(\mathcal{E O}),(\mathcal{O O})]_{+}^{2}+\frac{\beta_{2}}{8 m_{1}^{2} m_{2} c^{6}}[(\mathcal{O E}),(\mathcal{O O})]_{+}^{2}- \\
& -\frac{\beta_{1} m_{1}-\beta_{2} m_{2}}{16 m_{1}^{2}\left(m_{1}^{2}-m_{2}^{2}\right) c^{6}}\left[(\mathcal{O O}),\left[(\mathcal{O E}),[(\mathcal{O E}),(\mathcal{O O})]_{+}\right]_{+}\right]_{+}- \\
& -\frac{\beta_{1} m_{1}-\beta_{2} m_{2}}{16 m_{2}^{2}\left(m_{1}^{2}-m_{2}^{2}\right) c^{6}}\left[(\mathcal{O O}),\left[(\mathcal{E O}),[(\mathcal{E O}),(\mathcal{O O})]_{+}\right]_{+}\right]_{+}- \\
& -\frac{\beta_{1}\left(\beta_{1} m_{1}-\beta_{2} m_{2}\right)^{2}}{16 m_{1}\left(m_{1}^{2}-m_{2}^{2}\right)^{2} c^{6}}\left[(\mathcal{O O}),\left[(\mathcal{O O}),(\mathcal{O E})^{2}\right]_{+}\right]_{+}- \\
& -\frac{\beta_{2}\left(\beta_{1} m_{1}-\beta_{2} m_{2}\right)^{2}}{16 m_{2}\left(m_{1}^{2}-m_{2}^{2}\right)^{2} c^{6}}\left[(\mathcal{O O}),\left[(\mathcal{O O}),(\mathcal{E O})^{2}\right]_{+}\right]_{+}- \\
& -\frac{\beta_{1} m_{2}-\beta_{2} m_{1}}{8 m_{1} m_{2}\left(m_{1}^{2}-m_{2}^{2}\right) c^{6}}[(\mathcal{O O}),[(\mathcal{O E}),[(\mathcal{E O}),(\mathcal{E E})]]]_{+}+ \\
& +\frac{\beta_{1}}{8 m_{1} m_{2}^{2} c^{6}}\left[[(\mathcal{E O}),(\mathcal{E E})],[(\mathcal{O E}),(\mathcal{O O})]_{+}\right]+ \\
& +\frac{\beta_{2}}{8 m_{1}^{2} m_{2} c^{6}}\left[[(\mathcal{O E}),(\mathcal{E E})],[(\mathcal{E O}),(\mathcal{O O})]_{+}\right]+ \\
& +\frac{\beta_{1} m_{2}-\beta_{2} m_{1}}{24 m_{1} m_{2}\left(m_{1}^{2}-m_{2}^{2}\right) c^{6}}[[(\mathcal{O E})(\mathcal{E O}),(\mathcal{O O})],(\mathcal{E E})]+ \\
& +\frac{\beta_{1} m_{2}+\beta_{2} m_{1}}{24 m_{1} m_{2}\left(m_{1}^{2}-m_{2}^{2}\right) c^{6}} \times \\
& \times[(\mathcal{E O})(\mathcal{O O})(\mathcal{O E})-(\mathcal{O E})(\mathcal{O O})(\mathcal{E O}),(\mathcal{E E})]+ \\
& +\frac{\left(\beta_{1} m_{1}-\beta_{2} m_{2}\right)^{2}}{8\left(m_{1}^{2}-m_{2}^{2}\right)^{2} c^{4}}[(\mathcal{O O}),[(\mathcal{E} \mathcal{E}),(\mathcal{O O})]] .
\end{aligned}
$$

The second type includes the higher-order terms that are linear in the original even-even and odd-odd terms and can be represented in the following way:

$$
H_{C}=\frac{1}{384 m_{1}^{4} c^{8}}[(\mathcal{O E}),[(\mathcal{O E}),[(\mathcal{O E}),[(\mathcal{O E}),(\mathcal{E E})]]]]+
$$




$$
\begin{aligned}
& +\frac{1}{12 m_{1}^{4} c^{8}}\left[(\mathcal{O E})^{3},[(\mathcal{O E}),(\mathcal{E} \mathcal{E})]\right]+ \\
& +\frac{1}{384 m_{2}^{4} c^{8}}[(\mathcal{E O}),[(\mathcal{E O}),[(\mathcal{E O}),[(\mathcal{E O}),(\mathcal{E} \mathcal{E})]]]]+
\end{aligned}
$$$$
+\frac{1}{12 m_{2}^{4} c^{8}}\left[(\mathcal{E O})^{3},[(\mathcal{E O}),(\mathcal{E} \mathcal{E})]\right]+
$$$$
+\frac{1}{64 m_{1}^{2} m_{2}^{2} c^{8}}[(\mathcal{O E}),[(\mathcal{O} \mathcal{E}),[(\mathcal{E O}),[(\mathcal{E O}),(\mathcal{E} \mathcal{E})]]]]-
$$

$$
\begin{aligned}
& -\frac{\beta_{1} \beta_{2}}{96 m_{1}^{3} m_{2} c^{8}}\left\{8\left[(\mathcal{O E})^{3},[(\mathcal{E O}),(\mathcal{O O})]_{+}\right]_{+}+\right. \\
& \left.+\left[(\mathcal{O E}),\left[(\mathcal{O E}),\left[(\mathcal{O E}),[(\mathcal{E O}),(\mathcal{O O})]_{+}\right]_{+}\right]_{+}\right]_{+}\right\}-
\end{aligned}
$$$$
-\frac{\beta_{1} \beta_{2}}{96 m_{1} m_{2}^{3} c^{8}}\left\{8\left[(\mathcal{O E}),\left[(\mathcal{E O})^{3},(\mathcal{O O})\right]_{+}\right]_{+}+\right.
$$$$
\left.+\left[(\mathcal{O E}),\left[(\mathcal{E O}),\left[(\mathcal{E O}),[(\mathcal{E O}),(\mathcal{O O})]_{+}\right]_{+}\right]_{+}\right]_{+}\right\}+
$$$$
+\frac{\beta_{1} m_{2}-\beta_{2} m_{1}}{48 m_{1} m_{2}\left(m_{1}^{2}-m_{2}^{2}\right) c^{8}} \times
$$$$
\times\left\{\frac{\beta_{1}}{m_{1}}\left[[(\mathcal{O E})(\mathcal{E O}),(\mathcal{O O})],(\mathcal{O E})^{2}\right]+\right.
$$$$
\left.+\frac{\beta_{2}}{m_{2}}\left[[(\mathcal{O E})(\mathcal{E O}),(\mathcal{O O})],(\mathcal{E O})^{2}\right]\right\}+
$$

$$
+\frac{\beta_{1} m_{2}+\beta_{2} m_{1}}{48 m_{1} m_{2}\left(m_{1}^{2}-m_{2}^{2}\right) c^{8}} \times
$$

$$
\times\left\{\frac{\beta_{1}}{m_{1}}\left[(\mathcal{E O})(\mathcal{O O})(\mathcal{O E})-(\mathcal{O E})(\mathcal{O O})(\mathcal{E O}),(\mathcal{O E})^{2}\right]+\right.
$$$$
\left.+\frac{\beta_{2}}{m_{2}}\left[(\mathcal{E O})(\mathcal{O O})(\mathcal{O E})-(\mathcal{O E})(\mathcal{O O})(\mathcal{E O}),(\mathcal{E O})^{2}\right]\right\}+
$$

$$
+\frac{\beta_{1}}{16 m_{1}^{5} c^{10}}(\mathcal{O E})^{6}+\frac{\beta_{2}}{16 m_{2}^{5} c^{10}}(\mathcal{E O})^{6} .
$$

$$
[(\mathcal{O} \mathcal{E}),(\mathcal{E O})]=0
$$

Here, as in the previous section, we assume that $(\mathcal{E} \mathcal{E})$, $(\mathcal{O O})$ are of order $c^{0}$, and $(\mathcal{O E}),(\mathcal{E O})$ are of order $c^{1}$. Expressions (9) have been obtained with the use of the generating functions (6) with regard for the commutative relation ond order, a considerable simplification of the fourthorder part of the transformed Hamiltonian. We note that it is necessary to do three iterations to obtain the transformed two-body Hamiltonian in the second and fourth orders. For the first iteration, one should limit oneself to the first seven terms $(n=6)$ in transformation (2). The third iteration only removes all the even-odd, odd-even, and odd-odd terms to the fourth order from the Hamiltonian without changing its even-even part.

We emphasize that $H_{B}$ and $H_{C}$ include all the terms of order $1 / c^{4}$ in the expansion. They together with the operator $H_{A}$ give the prescription of the transformation of the original Hamiltonian (5) into an even-even operator up to the fourth order:

$$
H_{\mathrm{tr}}=H_{A}+H_{B}+H_{C}+O\left(1 / c^{6}\right) .
$$

We call the sum $H_{B}+H_{C}$ representing the fourth-order part of $H_{\mathrm{tr}}$ as the higher-order transformed Hamiltonian. It should be noted that if the inverse mass is taken as the expansion parameter, all the terms of $H_{B}$ are of order $1 / m^{3}$ except term (9f) which is of order $1 / m^{2}$. In $H_{C}$, all the terms are of order $1 / \mathrm{m}^{4}$ except the last two ones which are of order $1 / \mathrm{m}^{5}$.

Actually, in contrast with $H_{A}$, the higher-order transformed Hamiltonian can be expressed in several different forms. Indeed, let us consider, for example, the first four terms of group $(9 \mathrm{~b})$. Using the relation

$$
\begin{aligned}
& {\left[(\mathcal{O O}),\left[(\mathcal{O E}),[(\mathcal{O E}),(\mathcal{O O})]_{+}\right]_{+}\right]_{+}=} \\
& =2[(\mathcal{O E}),(\mathcal{O O})]_{+}^{2}+\left[(\mathcal{O E}),\left[(\mathcal{O E}),(\mathcal{O O})^{2}\right]\right]
\end{aligned}
$$

we can rewrite them in such a manner:

$$
\begin{aligned}
& -\frac{\beta_{1} m_{2}-\beta_{2} m_{1}}{8 m_{1} m_{2}\left(m_{1}^{2}-m_{2}^{2}\right) c^{6}}[(\mathcal{O E}),(\mathcal{O O})]_{+}^{2}- \\
& -\frac{\beta_{1} m_{2}-\beta_{2} m_{1}}{8 m_{1} m_{2}\left(m_{1}^{2}-m_{2}^{2}\right) c^{6}}[(\mathcal{E O}),(\mathcal{O O})]_{+}^{2}- \\
& -\frac{\beta_{1} m_{1}-\beta_{2} m_{2}}{16 m_{1}^{2}\left(m_{1}^{2}-m_{2}^{2}\right) c^{6}}\left[(\mathcal{O E}),\left[(\mathcal{O E}),(\mathcal{O O})^{2}\right]\right]-
\end{aligned}
$$




$$
-\frac{\beta_{1} m_{1}-\beta_{2} m_{2}}{16 m_{2}^{2}\left(m_{1}^{2}-m_{2}^{2}\right) c^{6}}\left[(\mathcal{E O}),\left[(\mathcal{E O}),(\mathcal{O O})^{2}\right]\right]
$$

It is easy to see that there appeared other terms instead of the third and fourth terms of (9b), and the factors by the first two ones were also changed.

In the case where the mass of one particle becomes considerably great as compared with the mass of the other one, i.e. $m_{1} \rightarrow \infty$ or $m_{2} \rightarrow \infty$, expression (11) goes over into the corresponding expression (4) for a single Dirac particle in external fields. Under this condition, only terms $(9 \mathrm{a}, \mathrm{g}, \mathrm{l})$ remain in the higher-order transformed Hamiltonian and go over into the last four terms in (4); the rest of the commutators and anticommutators which involve the $(\mathcal{O O})$ terms in expressions (9) vanish. It is shown in the next section that, for the Breit equation, terms (9a, g) give relativistic corrections of the fourth order to the Coulomb interaction.

Though the expansion of a two-particle Hamiltonian to the second order gives an equation without any infinite terms, when both particles with equal masses are in positive or negative energy states (those correspond to setting $\beta_{1}=\beta_{2}= \pm 1$ ), one should remember, however, that expressions (8) and (9) are useful under condition that two particles have unequal masses, as the procedure applied here to convert the original Hamiltonian (5) into an even-even operator is correct only if $m_{1} \neq m_{2}$. Because of the structure of the function $S_{o o}$, the operators $H_{A}, H_{B}$, and $H_{C}$ involve certain terms having mass differences in the denominators if two particles are in different energy states $\left(\beta_{1}=1, \beta_{2}=-1\right.$, or $\beta_{1}=-1, \beta_{2}=1$ ) and, therefore, becoming infinite in the equal-mass case. Still, certain extra undesirable terms, with mass differences in their denominators, of another kind appear in the expansion of $H$ in higher orders. Indeed, the higher-order transformed Hamiltonian includes a new type of terms (see (9e) and $(9 \mathrm{k})$ ) having the differences of masses in the denominators, even although both particles are in a positive or negative energy state, and this type is not represented in the expansion to order $1 / c^{2}$. The terms with this structure enter, obviously, into the expansion also in higher orders. This is an important feature of the reduction of a two-body Hamiltonian to approximate forms of higher orders, which makes a difference from its expansion to the second order. So, each of the four parts of the total transformed Hamiltonian in higher orders involves undesirable terms becoming infinite when one puts $m_{1}=m_{2}$. The two parts of the Hamiltonian for both particles in a positive or negative energy state have mass differences in the denominators due to $(9 \mathrm{e}, \mathrm{k})$. The other two parts, for particles in different energy states, have mass differences due to the last four terms in (9b), the first term in $(9 \mathrm{c})$, and also $(9 \mathrm{~d}, \mathrm{j})$. In other words, even though one considers the most useful case of particles in positive energy states, when there are no mass differences in the denominators in the Hamiltonian reduced to order $1 / c^{2}$, there are infinite terms in higher orders in any case if $m_{1}=m_{2}$.

Yet, as it turns out, the undesirable terms $(9 \mathrm{e}, \mathrm{k})$ can be eliminated from the operators $H_{B}$ and $H_{C}$ provided that the transformed Hamiltonian has to be subjected to an additional canonical transformation like (2), where the function $S$ is a suitably chosen Hermitian operator. We represent it as an even-even operator of order $1 / c^{4}$ in the following form:

$$
\begin{aligned}
& S_{e e}=-\frac{i\left(\beta_{1} m_{2}+\beta_{2} m_{1}\right)}{24 m_{1} m_{2}\left(m_{1}^{2}-m_{2}^{2}\right) c^{6}} \times \\
& \times\{(\mathcal{O E})(\mathcal{O O})(\mathcal{E O})-(\mathcal{E O})(\mathcal{O O})(\mathcal{O} \mathcal{E})\} .
\end{aligned}
$$

Furthermore, taking its higher order into consideration, we may restrict ourselves to only the first two terms in transformation (2), and the rest of the terms can be omitted as they are of lower orders of magnitude,

$e^{i S_{e e}} H_{\mathrm{tr}} e^{-i S_{e e}} \approx H_{\mathrm{tr}}+i\left[S_{e e}, H_{\mathrm{tr}}\right]$.

Since the generating function $S_{e e}$ is an even-even operator, it commutes with the large terms of $H_{\mathrm{tr}}$ :

$\left[S_{e e}, \beta_{1} m_{1} c^{2}+\beta_{2} m_{2} c^{2}\right]=0$.

In fact, to remove undesired terms, it is quite convenient to retain the terms of order $c^{0}$ in $H_{\mathrm{tr}}$ that stand in the commutator of (15) and to discard the rest. So, we have

$$
\begin{aligned}
& i\left[S_{e e}, H_{\mathrm{tr}}\right] \approx i\left[S_{e e},(\mathcal{E} \mathcal{E})+\frac{\beta_{1}(\mathcal{O} \mathcal{E})^{2}}{2 m_{1} c^{2}}+\frac{\beta_{2}(\mathcal{E} \mathcal{O})^{2}}{2 m_{2} c^{2}}\right]= \\
& =-\frac{\beta_{1} m_{2}+\beta_{2} m_{1}}{24 m_{1} m_{2}\left(m_{1}^{2}-m_{2}^{2}\right) c^{6}} \times \\
& \times\{[(\mathcal{E O})(\mathcal{O O})(\mathcal{O E})-(\mathcal{O E})(\mathcal{O O})(\mathcal{E O}),(\mathcal{E} \mathcal{E})]+ \\
& +\frac{\beta_{1}}{2 m_{1} c^{2}}\left[(\mathcal{E} \mathcal{O})(\mathcal{O O})(\mathcal{O E})-(\mathcal{O E})(\mathcal{O O})(\mathcal{E O}),(\mathcal{O E})^{2}\right]+ \\
& \left.+\frac{\beta_{2}}{2 m_{2} c^{2}}\left[(\mathcal{E} \mathcal{O})(\mathcal{O O})(\mathcal{O E})-(\mathcal{O E})(\mathcal{O O})(\mathcal{E O}),(\mathcal{E O O})^{2}\right]\right\}
\end{aligned}
$$


which is just sufficient to eliminate $(9 \mathrm{e}, \mathrm{k})$ from the Hamiltonian. One should also pay attention to the distinction of the operator $S_{e e}$ from the generating functions (6).

Though $S_{e e}$ serves the similar purposes as the operators $S_{e o}, S_{o e}$, and $S_{o o}$ (to remove the undesirable terms from the Hamiltonian), it differs essentially from them. Thus, in contrast with $S_{e e}$, the main object of the prescription of the proper choice of (6) is to remove the even-odd, odd-even, and odd-odd terms from the twobody Hamiltonian, using the fact that they all do not commute with the large terms (see expressions (7)). On the other hand, the main purpose of the function $S_{e e}$ is to remove the undesirable even-even terms from the transformed even-even Hamiltonian, because it does not commute with the terms of the zeroth order in $H_{\mathrm{tr}}$. We remark that, while canceling the undesirable terms in expressions (9), this transformation gives rise, in general, to new undesirable even-even terms having mass differences in the denominators, but all of them are of lower orders of magnitude than the canceled ones. We also note that any of even-even operators, not only in the form (14), certainly satisfy relation (16).

Moreover, in addition to (14), there also exists another even-even function serving the similar object, but it only enables to remove terms $(9 \mathrm{~d}, \mathrm{j})$ and reads

$S_{e e}^{\prime}=-\frac{i\left(\beta_{1} m_{2}-\beta_{2} m_{1}\right)}{24 m_{1} m_{2}\left(m_{1}^{2}-m_{2}^{2}\right) c^{6}}[(\mathcal{O O}),(\mathcal{O E})(\mathcal{E O})]$

Indeed, one can easily verify that

$$
\begin{aligned}
& i\left[S_{e e}^{\prime}, H_{\mathrm{tr}}\right] \approx i\left[S_{e e}^{\prime},(\mathcal{E} \mathcal{E})+\frac{\beta_{1}(\mathcal{O} \mathcal{E})^{2}}{2 m_{1} c^{2}}+\frac{\beta_{2}(\mathcal{E O})^{2}}{2 m_{2} c^{2}}\right]= \\
& =-\frac{\beta_{1} m_{2}-\beta_{2} m_{1}}{24 m_{1} m_{2}\left(m_{1}^{2}-m_{2}^{2}\right) c^{6}}\{[(\mathcal{O} \mathcal{E})(\mathcal{E} \mathcal{O}),(\mathcal{O O})],(\mathcal{E} \mathcal{E})]+ \\
& +\frac{\beta_{1}}{2 m_{1} c^{2}}\left[[(\mathcal{O} \mathcal{E})(\mathcal{E O}),(\mathcal{O O})],(\mathcal{O} \mathcal{E})^{2}\right]+ \\
& \left.+\frac{\beta_{2}}{2 m_{2} c^{2}}\left[[(\mathcal{O} \mathcal{E})(\mathcal{E O}),(\mathcal{O O})],(\mathcal{E O})^{2}\right]\right\}
\end{aligned}
$$

We remark that $S_{e e}$ and $S_{e e}^{\prime}$ can be combined into one Hermitian even-even operator. Indeed, their sum forms another even-even operator which can be expressed in terms of $S_{e o}, S_{o e}$, and $S_{o o}$ in a convenient brief form:

$$
S_{e e}^{\prime \prime}=S_{e e}+S_{e e}^{\prime}=\frac{1}{3}\left[S_{e o},\left[S_{o e}, S_{o o}\right]\right]
$$

Introducing this function in expression (15) instead of $S_{e e}$, one can remove both terms $(9 \mathrm{e}, \mathrm{k})$ and $(9 \mathrm{~d}, \mathrm{j})$ from the higher-order transformed Hamiltonian.

Thus, the generating function (14) (or (18)) enables us to modify the transformed Hamiltonian, by eliminating all the fourth-order terms that have mass differences in the denominators (in the case of two particles in a positive or negative energy state) from it.

If the commutation relation (10) has not been taken into account, the form of the higher-order transformed two-body Hamiltonian would be more lengthy than we have in expressions (9). Thereto, the structure of the even-even operators $S_{e e}, S_{e e}^{\prime}$, and $S_{e e}^{\prime \prime}$ would be more awkward as well, than it is here. Furthermore, many new higher-order terms would also appear in the transformed Hamiltonian in expressions (9).

We note that, in contrast with the generating functions proposed in [6] which are also applicable to the case of equal masses of particles, the functions in the form (6) enable one to express the higher-order transformed Hamiltonian in a relatively simple form if relation (10) is satisfied.

\section{Application to the Breit Equation}

As a simple example, we consider the Breit equation for two Coulomb particles of charges $\epsilon_{1}$ and $\epsilon_{2}$, respectively:

$H \psi=\left\{c \boldsymbol{\alpha}_{1} \mathbf{p}_{1}+\beta_{1} m_{1} c^{2}+c \boldsymbol{\alpha}_{2} \mathbf{p}_{2}+\beta_{2} m_{2} c^{2}+\right.$

$\left.+\frac{\epsilon_{1} \epsilon_{2}}{r}-\frac{\epsilon_{1} \epsilon_{2}}{2 r}\left(\boldsymbol{\alpha}_{1} \boldsymbol{\alpha}_{2}+\frac{\left(\boldsymbol{\alpha}_{1} \mathbf{r}\right)\left(\boldsymbol{\alpha}_{2} \mathbf{r}\right)}{r^{2}}\right)\right\} \psi=E \psi$.

Here, $\mathbf{r}=\mathbf{r}_{1}-\mathbf{r}_{2}$ and $r=|\mathbf{r}|$. One ought to remember, however, that the total form of the original interaction in the equation can also include, in general, many other components in addition to the instantaneous Coulomb interaction and the Breit operator such as, for example, the intrinsic magnetic moment terms [7]. Nevertheless, we limit ourselves here to the treatment of the equation with the interaction in a simple form, as it is in (19).

Thus, for the Breit equation, we have

$$
\begin{aligned}
& (\mathcal{O} \mathcal{E})=c \boldsymbol{\alpha}_{1} \mathbf{p}_{1}, \quad(\mathcal{E O})=c \boldsymbol{\alpha}_{2} \mathbf{p}_{2}, \quad(\mathcal{E} \mathcal{E})=\frac{\epsilon_{1} \epsilon_{2}}{r} \\
& (\mathcal{O O})=-\frac{\epsilon_{1} \epsilon_{2}}{2 r}\left(\boldsymbol{\alpha}_{1} \boldsymbol{\alpha}_{2}+\frac{\left(\boldsymbol{\alpha}_{1} \mathbf{r}\right)\left(\boldsymbol{\alpha}_{2} \mathbf{r}\right)}{r^{2}}\right)
\end{aligned}
$$

One can see that, in this case, the $(\mathcal{E} \mathcal{E})$ and $(\mathcal{O O})$ terms denote the original interaction in $H$ and commute with 
each other. Applying (8), one can perform the expansion of $H$ to order $1 / c^{2}$ and, if $\beta_{1}=\beta_{2}=1$, get the Breit correction derived also in QED (see, e.g., [12,13]). Based on formula (8), the expansion of the Breit equation to the second order and the study of properties of the transformed Hamiltonian have been carried out in $[5,7]$. We note that the Breit correction is divergent, as it involves the Dirac $\delta$-functions appearing because of the Coulomb potential in $H$. Due to the same potential, the $\delta$-functions, already together with their derivatives, will also appear in the corrections of higher orders.

Since the expressions $H_{B}$ and $H_{C}$ are just suitable for the case of the Breit equation, it is convenient to work out the terms of (9) and thereby to obtain the higherorder Hamiltonian in an explicit form in the case under consideration. Below, with the use of notations (20), we calculate the $1 / c^{4}$-order terms in the expansion of Eq. (19), what is the main object of this section.

We start with two terms (9l)

$$
\frac{\beta_{1}}{16 m_{1}^{5} c^{10}}(\mathcal{O} \mathcal{E})^{6}+\frac{\beta_{2}}{16 m_{2}^{5} c^{10}}(\mathcal{E O})^{6}=\sum_{n=1,2} \frac{\beta_{n} \mathbf{p}_{n}^{6}}{16 m_{n}^{5} c^{4}},
$$

which yield, under condition that both particles are in positive energy states, the correction of order $1 / c^{4}$ to the kinetic energy. The rest of the components of (9) form the fourth-order relativistic corrections to the effective potential, which can be divided into three parts with respect to the contribution of $(\mathcal{E} \mathcal{E})$ and $(\mathcal{O O})$ to it.

The terms of the first part give a correction of the fourth order to the Coulomb interaction between two fermions. They are represented by $(9 \mathrm{a}, \mathrm{g}, \mathrm{h})$. For terms $(9 \mathrm{a})$, after substituting the operators $(\mathcal{O} \mathcal{E}),(\mathcal{E} \mathcal{O})$, and $(\mathcal{E} \mathcal{E})$ from $(20 \mathrm{a})$, one gets $(\hbar=1)$

$$
\begin{aligned}
& -\frac{\beta_{1}}{8 m_{1}^{3} c^{6}}[(\mathcal{O} \mathcal{E}),(\mathcal{E} \mathcal{E})]^{2}-\frac{\beta_{2}}{8 m_{2}^{3} c^{6}}[(\mathcal{E} \mathcal{O}),(\mathcal{E} \mathcal{E})]^{2}= \\
& =-\iint \frac{d^{3} q d^{3} k}{(2 \pi)^{6}}\left(\frac{\beta_{1}}{m_{1}^{3}}+\frac{\beta_{2}}{m_{2}^{3}}\right) \frac{\left(4 \pi \epsilon_{1} \epsilon_{2}\right)^{2}}{8 c^{4} \mathbf{q}^{2} \mathbf{k}^{2}} \mathbf{q k} e^{i(\mathbf{q}+\mathbf{k}) \mathbf{r}}= \\
& =\left(\frac{\beta_{1}}{m_{1}^{3}}+\frac{\beta_{2}}{m_{2}^{3}}\right) \frac{\left(\epsilon_{1} \epsilon_{2}\right)^{2}}{8 c^{4} r^{4}}
\end{aligned}
$$

In order not to miss any $\delta$-functions in the final results, it is convenient, in general, to work out the terms from $H_{B}$ and $H_{C}$ first in the momentum space and then to pass into the coordinate space, like the computation of corrections to the second order. Thus, the integration element in (22) contains the terms written in the momentum representation.

The sum of the first two terms of group (9g) can be presented as follows:

$$
\begin{aligned}
& \frac{1}{384 m_{1}^{4} c^{8}}[(\mathcal{O} \mathcal{E}),[(\mathcal{O E}),[(\mathcal{O E}),[(\mathcal{O} \mathcal{E}),(\mathcal{E} \mathcal{E})]]]]+ \\
& +\frac{1}{12 m_{1}^{4} c^{8}}\left[(\mathcal{O} \mathcal{E})^{3},[(\mathcal{O} \mathcal{E}),(\mathcal{E} \mathcal{E})]\right]= \\
& =\frac{1}{384 m_{1}^{4} c^{4}} \int \frac{d^{3} q}{(2 \pi)^{3}} \frac{4 \pi \epsilon_{1} \epsilon_{2}}{\mathbf{q}^{2}}\left\{9\left(\mathbf{q}^{2}+\left(2 \mathbf{p}_{1}-\mathbf{q}\right)^{2}\right) \times\right. \\
& \left.\times\left(\mathbf{q}^{2}-2 i \boldsymbol{\sigma}_{1}\left[\mathbf{q} \mathbf{p}_{1}\right]\right)+15\left(\mathbf{q}\left(2 \mathbf{p}_{1}-\mathbf{q}\right)\right)^{2}\right\} e^{i \mathbf{q r}}= \\
& =\frac{3 \epsilon_{1} \epsilon_{2}}{16 m_{1}^{4} c^{4}}\left\{\pi\left[\mathbf{p}_{1}^{2}, \delta(\mathbf{r})\right]+-2 \pi\left[\nabla \delta(\mathbf{r}) \mathbf{p}_{1}\right] \boldsymbol{\sigma}_{1}+\frac{\left[\mathbf{r} \mathbf{p}_{1}\right] \boldsymbol{\sigma}_{1}}{r^{3}} \mathbf{p}_{1}^{2}+\right. \\
& \left.+3 i r^{i} \frac{\left.\mathbf{r}_{1}\right]}{r^{5}} p_{1}^{i}\right\}+\frac{5}{128 m_{1}^{4} c^{4}}\left[\mathbf{p}_{1}^{2},\left[\mathbf{p}_{1}^{2}, \frac{\epsilon_{1} \epsilon_{2}}{r}\right]\right]
\end{aligned}
$$

Here, $[\mathbf{a b}]$ denotes the vector product of the vectors $\mathbf{a}$ and $\mathbf{b}$. The second component can be developed as

$$
\left[\mathbf{p}_{1}^{2},\left[\mathbf{p}_{1}^{2}, \frac{\epsilon_{1} \epsilon_{2}}{r}\right]\right]=\epsilon_{1} \epsilon_{2}\left\{-4 \pi \Delta \delta(\mathbf{r})-16 \pi i \nabla \delta(\mathbf{r}) \mathbf{p}_{1}+\right.
$$

$$
\left.+4\left[\frac{4 \pi}{3} \delta(\mathbf{r}) \delta^{i j}+\frac{1}{r^{3}}\left(\delta^{i j}-3 \frac{r^{i} r^{j}}{r^{2}}\right)\right] p_{1}^{i} p_{1}^{j}\right\} .
$$

The next two terms in expression $(9 \mathrm{~g})$ are symmetric to those derived above and can be obtained from them by interchanging indices 1,2 and by replacing $\mathbf{p}_{1}$ by $-\mathbf{p}_{2}$.

Then term (9h) yields

$$
\begin{aligned}
& {[(\mathcal{O E}),[(\mathcal{O} \mathcal{E}),[(\mathcal{E O}),[(\mathcal{E O}),(\mathcal{E} \mathcal{E})]]]]=\frac{c^{4}}{(2 \pi)^{3}} \int d^{3} q \frac{4 \pi \epsilon_{1} \epsilon_{2}}{\mathbf{q}^{2}}\left(\mathbf{q}^{2}-2 i \boldsymbol{\sigma}_{1}\left[\mathbf{q p}_{1}\right]\right)\left(\mathbf{q}^{2}+2 i \boldsymbol{\sigma}_{2}\left[\mathbf{q} \mathbf{p}_{2}\right]\right) e^{i \mathbf{q r}}=} \\
& =\epsilon_{1} \epsilon_{2} c^{4}\left\{-4 \pi \Delta \delta(\mathbf{r})-8 \pi\left[\nabla \delta(\mathbf{r}) \mathbf{p}_{1}\right] \boldsymbol{\sigma}_{1}+8 \pi\left[\nabla \delta(\mathbf{r}) \mathbf{p}_{2}\right] \boldsymbol{\sigma}_{2}+4\left[\frac{4 \pi}{3} \delta(\mathbf{r}) \delta^{i j}+\frac{1}{r^{3}}\left(\delta^{i j}-3 \frac{r^{i} r^{j}}{r^{2}}\right)\right]\left[\boldsymbol{\sigma}_{1} \mathbf{p}_{1}\right]^{i}\left[\boldsymbol{\sigma}_{2} \mathbf{p}_{2}\right]^{j}\right\}
\end{aligned}
$$


All these expressions, together with the terms symmetric to equation (23), represent the relativistic corrections of order $1 / c^{4}$ to the Coulomb interaction in the higher-order effective Hamiltonian. With neglect of the Breit operator (20b) in Eq. (19), only the expressions calculated above form the higher-order transformed Hamiltonian which can be also applicable to the equal-mass case. One can easily see that there are the singular operators involving the Dirac $\delta$-function and its derivatives in the treated terms. As it was emphasized, such singular operators appear there because of the Coulomb term represented by the even-even operator in $H$.

The second part of the terms is a little larger than the preceding one, comes from groups (9b, i, j, k), and describes the correction that is conditioned by the Breit operator, namely by the $(\mathcal{O O})$ terms. The computation of the second term from group $(9 \mathrm{~b})$ gives

$$
\begin{aligned}
& {[(\mathcal{O E}),(\mathcal{O O})]_{+}^{2}=\iint \frac{d^{3} q d^{3} k}{(2 \pi)^{6}} \frac{\left(4 \pi \epsilon_{1} \epsilon_{2}\right)^{2} c^{2}}{\mathbf{q}^{2} \mathbf{k}^{2}}\left(\delta^{i j}-\frac{q^{i} q^{j}}{\mathbf{q}^{2}}\right)\left(\delta^{\alpha \beta}-\frac{k^{\alpha} k^{\beta}}{\mathbf{k}^{2}}\right)\left\{2\left(2 p_{1}^{i}+i\left[\boldsymbol{\sigma}_{1} \mathbf{q}\right]^{i}\right)\left(p_{1}^{\alpha}-q^{\alpha}\right)+k^{i} q^{\alpha}-\mathbf{q} \mathbf{k} \delta^{i \alpha}+\right.} \\
& \left.+2 i p_{1}^{i}\left[\boldsymbol{\sigma}_{1} \mathbf{k}\right]^{\alpha}-i[\mathbf{q k}]^{i} \sigma_{1}^{\alpha}-i \varepsilon^{m i \alpha} q^{m}\left(\boldsymbol{\sigma}_{1} \mathbf{k}\right)\right\}\left(\delta^{j \beta}+i \varepsilon^{j \beta k} \sigma_{2}^{k}\right) e^{i(\mathbf{q}+\mathbf{k}) \mathbf{r}}=\frac{\left(\epsilon_{1} \epsilon_{2}\right)^{2} c^{2}}{r}\left\{\frac{2}{r^{3}}-\frac{2}{r^{3}}\left[\mathbf{r} \mathbf{p}_{1}\right] \boldsymbol{\sigma}_{1}-\frac{3}{r^{3}}\left[\mathbf{r} \mathbf{p}_{1}\right] \boldsymbol{\sigma}_{2}-\right. \\
& \left.-\frac{18}{r^{3}} \boldsymbol{\sigma}_{1} \boldsymbol{\sigma}_{2}+\left(\frac{7}{r^{3}}-\frac{4 \pi}{3} \delta(\mathbf{r})\right)\left(3 \delta^{i j}-\frac{r^{i} r^{j}}{r^{2}}\right) \sigma_{1}^{i} \sigma_{2}^{j}+\frac{1}{r}\left(\delta^{i j}+3 \frac{r^{i} r^{j}}{r^{2}}\right) p_{1}^{i} p_{1}^{j}+\frac{2 i}{r^{3}} \mathbf{r p}_{1}\right\}
\end{aligned}
$$

where $\varepsilon^{a b c}$ is the unit absolutely antisymmetric tensor. Instead of the straightforward calculation of the next type of the terms from (9b), we can apply Eq. (12) where the first term standing on the right-hand side is already worked out in (25), and the second term is simpler than the one on the left-hand side. The computation of it yields

$$
\begin{aligned}
& {\left[(\mathcal{O E}),\left[(\mathcal{O} \mathcal{E}),(\mathcal{O O})^{2}\right]\right]=\iint \frac{d^{3} q d^{3} k}{(2 \pi)^{6}} \frac{\left(4 \pi \epsilon_{1} \epsilon_{2}\right)^{2} c^{2}}{\mathbf{q}^{2} \mathbf{k}^{2}}\left(\delta^{i j}-\frac{q^{i} q^{j}}{\mathbf{q}^{2}}\right)\left(\delta^{\alpha \beta}-\frac{k^{\alpha} k^{\beta}}{\mathbf{k}^{2}}\right)\left\{2\left(\mathbf{q}(\mathbf{q}+\mathbf{k})-2 i \boldsymbol{\sigma}_{1}\left[\mathbf{q} \mathbf{p}_{1}\right]\right) \delta^{i \alpha} \delta^{j \beta}+\right.} \\
& \left.+4 i q^{\alpha} p_{1}^{i} \varepsilon^{j \beta n} \sigma_{2}^{n}-\left(2 \mathbf{p}_{1}-\mathbf{q}-\mathbf{k}\right)^{2} \varepsilon^{i \alpha m} \varepsilon^{j \beta n} \sigma_{1}^{m} \sigma_{2}^{n}+4\left(p_{1}^{k} p_{1}^{l}-p_{1}^{k} q^{l}-q^{k} p_{1}^{l}\right) \varepsilon^{i \alpha k} \varepsilon^{j \beta n} \sigma_{1}^{l} \sigma_{2}^{n}\right\} e^{i(\mathbf{q}+\mathbf{k}) \mathbf{r}}= \\
& =\frac{\left(\epsilon_{1} \epsilon_{2}\right)^{2} c^{2}}{r}\left\{-\frac{3}{r^{3}}+\frac{32 \pi}{3} \delta(\mathbf{r})+\frac{6}{r^{3}}\left[\mathbf{r p}_{1}\right] \boldsymbol{\sigma}_{1}+\frac{3}{r^{3}}\left[\mathbf{r} \mathbf{p}_{1}\right] \boldsymbol{\sigma}_{2}+\frac{7}{r^{3}} \boldsymbol{\sigma}_{1} \boldsymbol{\sigma}_{2}-2\left(\frac{1}{r^{3}}+\frac{4 \pi}{3} \delta(\mathbf{r})\right)\left(3 \delta^{i j}-\frac{r^{i} r^{j}}{r^{2}}\right) \sigma_{1}^{i} \sigma_{2}^{j}-\right. \\
& \left.-\frac{2}{r}\left(2 \delta^{i j}-\frac{r^{i} r^{j}}{r^{2}}\right)\left(\sigma_{1}^{i} \sigma_{2}^{j} \mathbf{p}_{1}^{2}-\left(\boldsymbol{\sigma}_{1} \mathbf{p}_{1}\right) \sigma_{2}^{i} p_{1}^{j}\right)+\frac{i}{r^{3}}\left[2 r^{i} p_{1}^{j}+3 r^{j} p_{1}^{i}-\left(7 \delta^{i j}-4 \frac{r^{i} r^{j}}{r^{2}}\right) \mathbf{r p}_{1}\right] \sigma_{1}^{i} \sigma_{2}^{j}\right\} .
\end{aligned}
$$

Then, for the last type of the anticommutators from group (9b), we have

$$
\begin{aligned}
& {\left[(\mathcal{O O}),\left[(\mathcal{O O}),(\mathcal{O} \mathcal{E})^{2}\right]_{+}\right]_{+}=\iint \frac{d^{3} q d^{3} k}{(2 \pi)^{6}} \frac{\left(4 \pi \epsilon_{1} \epsilon_{2}\right)^{2} c^{2}}{\mathbf{q}^{2} \mathbf{k}^{2}}\left(\delta^{i j}-\frac{q^{i} q^{j}}{\mathbf{q}^{2}}\right)\left(\delta^{\alpha \beta}-\frac{k^{\alpha} k^{\beta}}{\mathbf{k}^{2}}\right)\left\{\left(2 \mathbf{q}^{2}+\left(2 \mathbf{p}_{1}-\mathbf{q}-\mathbf{k}\right)^{2}\right) \times\right.} \\
& \left.\times\left(\delta^{i \alpha} \delta^{j \beta}-\varepsilon^{i \alpha m} \varepsilon^{j \beta n} \sigma_{1}^{m} \sigma_{2}^{n}\right)-2 \mathbf{q}\left(2 \mathbf{p}_{1}-\mathbf{q}\right)\left(i \delta^{j \beta} \varepsilon^{i \alpha m} \sigma_{1}^{m}+i \delta^{i \alpha} \varepsilon^{j \beta n} \sigma_{2}^{n}\right)\right\} e^{i(\mathbf{q}+\mathbf{k}) \mathbf{r}}=
\end{aligned}
$$




$$
\begin{aligned}
& =\frac{\left(\epsilon_{1} \epsilon_{2}\right)^{2} c^{2}}{r}\left\{-\frac{1}{r^{3}}+\frac{64 \pi}{3} \delta(\mathbf{r})+\frac{1}{r^{3}}\left[\mathbf{r p}_{1}\right]\left(\boldsymbol{\sigma}_{1}+\boldsymbol{\sigma}_{2}\right)+\frac{13}{r^{3}} \boldsymbol{\sigma}_{1} \boldsymbol{\sigma}_{2}-4\left(\frac{1}{r^{3}}+\frac{4 \pi}{3} \delta(\mathbf{r})\right)\left(3 \delta^{i j}-\frac{r^{i} r^{j}}{r^{2}}\right) \sigma_{1}^{i} \sigma_{2}^{j}+\right. \\
& \left.+\frac{2}{r}\left[3-\left(2 \delta^{i j}-\frac{r^{i} r^{j}}{r^{2}}\right) \sigma_{1}^{i} \sigma_{2}^{j}\right] \mathbf{p}_{1}^{2}+\frac{12 i}{r^{3}} \mathbf{r p}_{1}-\frac{2 i}{r^{3}}\left[r^{i} p_{1}^{j}+r^{j} p_{1}^{i}+4\left(\delta^{i j}-\frac{r^{i} r^{j}}{r^{2}}\right) \mathbf{r} \mathbf{p}_{1}\right] \sigma_{1}^{i} \sigma_{2}^{j}\right\}
\end{aligned}
$$

The two terms in the first brackets in group (9i) can be represented as

$$
\begin{aligned}
& 8\left[(\mathcal{O} \mathcal{E})^{3},[(\mathcal{E O}),(\mathcal{O O})]_{+}\right]_{+}+\left[(\mathcal{O} \mathcal{E}),\left[(\mathcal{O} \mathcal{E}),\left[(\mathcal{O E}),[(\mathcal{E O}),(\mathcal{O O})]_{+}\right]_{+}\right]_{+}\right]_{+}=-\frac{3 c^{4}}{(2 \pi)^{3}} \int d^{3} q \frac{4 \pi \epsilon_{1} \epsilon_{2}}{\mathbf{q}^{2}}\left(\delta^{i j}-\frac{q^{i} q^{j}}{\mathbf{q}^{2}}\right) \times \\
& \times\left\{\left(\mathbf{q}^{2}+\left(2 \mathbf{p}_{1}-\mathbf{q}\right)^{2}\right)\left(2 p_{1}^{i}+i\left[\boldsymbol{\sigma}_{1} \mathbf{q}\right]^{i}\right)+i \mathbf{q}\left(2 \mathbf{p}_{1}-\mathbf{q}\right)\left[\boldsymbol{\sigma}_{1}\left(2 \mathbf{p}_{1}-\mathbf{q}\right)\right]^{i}\right\}\left(2 p_{2}^{j}-i\left[\boldsymbol{\sigma}_{2} \mathbf{q}\right]^{j}\right) e^{i \mathbf{q r}}= \\
& =-\epsilon_{1} \epsilon_{2} c^{4}\left(2 p_{2}^{j}-\left[\boldsymbol{\sigma}_{2} \nabla\right]^{j}\right)\left\{\left(2 p_{1}^{i}+\left[\boldsymbol{\sigma}_{1} \nabla\right]^{i}\right)\left[\mathbf{p}_{1}^{2}, \frac{3}{r}\left(\delta^{i j}+\frac{r^{i} r^{j}}{r^{2}}\right)\right]_{+}+\left[\boldsymbol{\sigma}_{1}\left(2 i \mathbf{p}_{1}-\nabla\right)\right]^{i}\left[\mathbf{p}_{1}^{2}, \frac{3}{2 r}\left(\delta^{i j}+\frac{r^{i} r^{j}}{r^{2}}\right)\right]\right\} .
\end{aligned}
$$

The next two terms of (9i) are symmetric to the ones calculated in (28). Then, for (9j), we write

$$
\begin{aligned}
& {\left[[(\mathcal{O} \mathcal{E})(\mathcal{E O}),(\mathcal{O O})],(\mathcal{O} \mathcal{E})^{2}\right]=\frac{i c^{4}}{2(2 \pi)^{3}} \int d^{3} q \frac{4 \pi \epsilon_{1} \epsilon_{2}}{\mathbf{q}^{2}}\left(\delta^{i j}-\frac{q^{i} q^{j}}{\mathbf{q}^{2}}\right) \mathbf{q}\left(2 \mathbf{p}_{1}-\mathbf{q}\right)\left\{\left[\boldsymbol{\sigma}_{1}\left(2 \mathbf{p}_{1}-\mathbf{q}\right)\right]^{i}\left(2 p_{2}^{j}-i\left[\boldsymbol{\sigma}_{2} \mathbf{q}\right]^{j}\right)+\right.} \\
& \left.+\left(2 p_{1}^{i}+i\left[\boldsymbol{\sigma}_{1} \mathbf{q}\right]^{i}\right)\left[\boldsymbol{\sigma}_{2}\left(2 \mathbf{p}_{2}+\mathbf{q}\right)\right]^{j}\right\} e^{i \mathbf{q r}}=c^{4}\left[\boldsymbol{\sigma}_{1}\left(2 i \mathbf{p}_{1}-\nabla\right)\right]^{i}\left(2 p_{2}^{j}-\left[\boldsymbol{\sigma}_{2} \nabla\right]^{j}\right)\left[\mathbf{p}_{1}^{2}, \frac{\epsilon_{1} \epsilon_{2}}{4 r}\left(\delta^{i j}+\frac{r^{i} r^{j}}{r^{2}}\right)\right]+ \\
& +c^{4}\left(2 p_{1}^{i}+\left[\boldsymbol{\sigma}_{1} \nabla\right]^{i}\right)\left[\boldsymbol{\sigma}_{2}\left(2 i \mathbf{p}_{2}+\nabla\right)\right]^{j}\left[\mathbf{p}_{1}^{2}, \frac{\epsilon_{1} \epsilon_{2}}{4 r}\left(\delta^{i j}+\frac{r^{i} r^{j}}{r^{2}}\right)\right] .
\end{aligned}
$$

Finally, we compute the first commutator in (9k) as follows:

$$
\begin{aligned}
& {\left[(\mathcal{E O})(\mathcal{O O})(\mathcal{O} \mathcal{E})-(\mathcal{O} \mathcal{E})(\mathcal{O O})(\mathcal{E O}),(\mathcal{O} \mathcal{E})^{2}\right]=-\frac{i c^{4}}{2(2 \pi)^{3}} \int d^{3} q \frac{4 \pi \epsilon_{1} \epsilon_{2}}{\mathbf{q}^{2}}\left(\delta^{i j}-\frac{q^{i} q^{j}}{\mathbf{q}^{2}}\right) \mathbf{q}\left(2 \mathbf{p}_{1}-\mathbf{q}\right)\left\{\left[\boldsymbol{\sigma}_{1}\left(2 \mathbf{p}_{1}-\mathbf{q}\right)\right]^{i} \times\right.} \\
& \left.\times\left(2 p_{2}^{j}-i\left[\boldsymbol{\sigma}_{2} \mathbf{q}\right]^{j}\right)-\left(2 p_{1}^{i}+i\left[\boldsymbol{\sigma}_{1} \mathbf{q}\right]^{i}\right)\left[\boldsymbol{\sigma}_{2}\left(2 \mathbf{p}_{2}+\mathbf{q}\right)\right]^{j}\right\} e^{i \mathbf{q r}}=-c^{4}\left[\boldsymbol{\sigma}_{1}\left(2 i \mathbf{p}_{1}-\nabla\right)\right]^{i}\left(2 p_{2}^{j}-\left[\boldsymbol{\sigma}_{2} \nabla\right]^{j}\right)\left[\mathbf{p}_{1}^{2}, \frac{\epsilon_{1} \epsilon_{2}}{4 r}\left(\delta^{i j}+\frac{r^{i} r^{j}}{r^{2}}\right)\right]+ \\
& +c^{4}\left(2 p_{1}^{i}+\left[\boldsymbol{\sigma}_{1} \nabla\right]^{i}\right)\left[\boldsymbol{\sigma}_{2}\left(2 i \mathbf{p}_{2}+\nabla\right)\right]^{j}\left[\mathbf{p}_{1}^{2}, \frac{\epsilon_{1} \epsilon_{2}}{4 r}\left(\delta^{i j}+\frac{r^{i} r^{j}}{r^{2}}\right)\right]
\end{aligned}
$$

Notice that expressions (29) and (30) are similar to each other except for the sign of the first term. As for the rest of the terms from groups (9b) and $(9 \mathrm{i}, \mathrm{j}, \mathrm{k})$, they are symmetric to the calculated ones.
Since commutators (9a, g, h) involve operators (20a) only, the calculations of them lead to relatively simple expressions. However, as it is easy to see, the terms from group (9b) have more lengthy forms than the ones in (22), (23), and (24). The terms computed in (25)-30) 
have a more awkward structure than the others in (9), as the terms of $(9 \mathrm{~b}, \mathrm{i}, \mathrm{j}, \mathrm{k})$ involve six $\sigma$-matrices, which leads to tedious calculations.

The third part of the corrections of order $1 / c^{4}$ comes from terms $(9 \mathrm{c}, \mathrm{d}, \mathrm{e}, \mathrm{f})$. They all involve the $(\mathcal{E} \mathcal{E})$ and
$(\mathcal{O O})$ terms and, therefore, give the contribution conditioned by both the static Coulomb interaction and the Breit operator. These are the terms nonlinear in the original interaction. The computation of the first term in $(9 \mathrm{c})$ yields

$[(\mathcal{O O}),[(\mathcal{O E}),[(\mathcal{E O}),(\mathcal{E} \mathcal{E})]]]_{+}=\frac{2 c^{2}}{(2 \pi)^{6}} \iint d^{3} q d^{3} k \frac{4 \pi \epsilon_{1} \epsilon_{2}}{\mathbf{q}^{2}} \frac{4 \pi \epsilon_{1} \epsilon_{2}}{\mathbf{k}^{2}}\left(\delta^{i j}-\frac{k^{i} k^{j}}{\mathbf{k}^{2}}\right)\left\{q^{i} q^{j}-\left[\boldsymbol{\sigma}_{1} \mathbf{q}\right]^{i}\left[\boldsymbol{\sigma}_{2} \mathbf{q}\right]^{j}\right\} e^{i(\mathbf{q}+\mathbf{k}) \mathbf{r}}=$

$=-\frac{2\left(\epsilon_{1} \epsilon_{2}\right)^{2} c^{2}}{r}\left\{\frac{1}{r^{3}}-\frac{8 \pi}{3} \delta(\mathbf{r})+\frac{3}{r^{3}} \boldsymbol{\sigma}_{1} \boldsymbol{\sigma}_{2}-\left(\frac{1}{r^{3}}-\frac{2 \pi}{3} \delta(\mathbf{r})\right)\left(3 \delta^{i j}-\frac{r^{i} r^{j}}{r^{2}}\right) \sigma_{1}^{i} \sigma_{2}^{j}\right\}$.

The expression for the second term of (9c) takes the form

$$
\begin{aligned}
& {\left[[(\mathcal{E} \mathcal{O}),(\mathcal{E} \mathcal{E})],[(\mathcal{O} \mathcal{E}),(\mathcal{O O})]_{+}\right]=-\frac{2 c^{2}}{(2 \pi)^{6}} \iint d^{3} q d^{3} k \frac{4 \pi \epsilon_{1} \epsilon_{2}}{\mathbf{q}^{2}} \frac{4 \pi \epsilon_{1} \epsilon_{2}}{\mathbf{k}^{2}}\left(\delta^{i j}-\frac{k^{i} k^{j}}{\mathbf{k}^{2}}\right)\left\{q^{i} q^{j}-i\left(2 p_{1}^{i}-q^{i}\right)\left[\boldsymbol{\sigma}_{2} \mathbf{q}\right]^{j}+\right.} \\
& \left.+\left[\boldsymbol{\sigma}_{1} \mathbf{k}\right]^{i}\left[\boldsymbol{\sigma}_{2} \mathbf{q}\right]^{j}\right\} e^{i(\mathbf{q}+\mathbf{k}) \mathbf{r}}=\frac{2\left(\epsilon_{1} \epsilon_{2}\right)^{2} c^{2}}{r}\left\{\frac{1}{r^{3}}-\frac{8 \pi}{3} \delta(\mathbf{r})-\frac{1}{r^{3}}\left[\mathbf{r p}_{1}\right] \boldsymbol{\sigma}_{2}+\frac{1}{r^{3}}\left(\delta^{i j}-\frac{r^{i} r^{j}}{r^{2}}\right) \sigma_{1}^{i} \sigma_{2}^{j}\right\} .
\end{aligned}
$$

Finally, we have obtained the following expressions for commutators $(9 \mathrm{~d})$ and $(9 \mathrm{e})$ :

$$
\begin{aligned}
& {[[(\mathcal{O} \mathcal{E})(\mathcal{E O}),(\mathcal{O O})],(\mathcal{E} \mathcal{E})]=} \\
& =-\frac{c^{2}}{(2 \pi)^{6}} \iint d^{3} q d^{3} k \frac{4 \pi \epsilon_{1} \epsilon_{2}}{\mathbf{q}^{2}} \frac{4 \pi \epsilon_{1} \epsilon_{2}}{\mathbf{k}^{2}}\left(\delta^{i j}-\frac{k^{i} k^{j}}{\mathbf{k}^{2}}\right) \times \\
& \times\left\{-i q^{i}\left[\boldsymbol{\sigma}_{1}\left(2 \mathbf{p}_{1}-\mathbf{q}\right)\right]^{j}+i\left(2 p_{2}^{i}+q^{i}\right)\left[\boldsymbol{\sigma}_{1} \mathbf{q}\right]^{j}-\right. \\
& -i\left(2 p_{1}^{i}-q^{i}\right)\left[\boldsymbol{\sigma}_{2} \mathbf{q}\right]^{j}+i q^{i}\left[\boldsymbol{\sigma}_{2}\left(2 \mathbf{p}_{2}+\mathbf{q}\right)\right]^{j}+ \\
& \left.+\left[\boldsymbol{\sigma}_{1} \mathbf{q}\right]^{i}\left[\boldsymbol{\sigma}_{2} \mathbf{k}\right]^{j}+\left[\boldsymbol{\sigma}_{1} \mathbf{k}\right]^{i}\left[\boldsymbol{\sigma}_{2} \mathbf{q}\right]^{j}\right\} e^{i(\mathbf{q}+\mathbf{k}) \mathbf{r}}= \\
& =\frac{\left(\epsilon_{1} \epsilon_{2}\right)^{2} c^{2}}{r^{4}}\left\{\left[\mathbf{r} \mathbf{p}_{1}\right]\left(2 \boldsymbol{\sigma}_{1}-\boldsymbol{\sigma}_{2}\right)+\left[\mathbf{r} \mathbf{p}_{2}\right]\left(\boldsymbol{\sigma}_{1}-2 \boldsymbol{\sigma}_{2}\right)+\right. \\
& \left.+2\left(\delta^{i j}-\frac{r^{i} r^{j}}{r^{2}}\right) \sigma_{1}^{i} \sigma_{2}^{j}\right\} \\
& {[(\mathcal{E O})(\mathcal{O O})(\mathcal{O} \mathcal{E})-(\mathcal{O} \mathcal{E})(\mathcal{O O})(\mathcal{E} \mathcal{O}),(\mathcal{E} \mathcal{E})]=}
\end{aligned}
$$

So far as term (9f) is concerned, it is equal to zero, because $(\mathcal{E} \mathcal{E})$ and $(\mathcal{O O})$ commute with each other in the case of the Breit equation. Note that all of the terms in the third part are only spin-depended ones.

As was noted, expressions (8) and (9) can be applicable to expand the Breit equation to the fourth order only if $m_{1} \neq m_{2}$. In this case, both the procedure of expressing the small components of the spinor $\psi$ in terms of its large components, applied to $H$ to get its expansion to order $1 / c^{2}$, and expression (8) lead to the same results. However, we cannot assert that this occurs in higher orders. In other words, we cannot state that all the terms of (9) with (22)-(34) agree 
with the terms derived by the method of large components. Thus, obtaining terms $(9 \mathrm{e}, \mathrm{k})$ within the method of large components is open to question. Otherwise, the expansion of the Breit equation to order $1 / c^{4}$ cannot be performed in the equal-mass case in principle, even if $\beta_{1}=\beta_{2}= \pm 1$. The presence of the terms with mass differences in the denominators in (8) is conditioned by the structure of the Breit equation which cannot be reduced in the case of particles in different energy states if $m_{1}=m_{2}$. On the other hand, the appearance of the terms having mass differences in the denominators in (9) when both particles are in a positive or negative energy state is probably conditioned by choosing $S_{o o}$ in the form (6b).

We note that the higher-order part of the transformed Breit Hamiltonian which follows from (9) with regard for (21)-(34) can be easily modified. Indeed, as we have shown in Section 3, terms $(9 \mathrm{~d}, \mathrm{e}, \mathrm{j}, \mathrm{k})$ can be eliminated by applying the function $S_{e e}^{\prime \prime}$ and do not give a contribution to the self-energy. Still, this transformation is not the only one that modifies the $1 / c^{4}$-order Hamiltonian. Consider, for example, a Hermitian even-even operator in the form ${ }^{1}$

$S_{e e}=-\frac{i}{16 c^{6}}\left[\frac{\beta_{1}}{m_{1}^{3}}(\mathcal{O E})^{2}+\frac{\beta_{2}}{m_{2}^{3}}(\mathcal{E} \mathcal{O})^{2},(\mathcal{E} \mathcal{E})\right]$,

where we bear in mind that $(\mathcal{O E}),(\mathcal{E} \mathcal{O})$, and $(\mathcal{E} \mathcal{E})$ are defined by (20a). Under this condition, operator (35) reads

$S_{e e}=-\frac{i}{16 c^{4}}\left[\frac{\beta_{1}}{m_{1}^{3}} \mathbf{p}_{1}^{2}+\frac{\beta_{2}}{m_{2}^{3}} \mathbf{p}_{2}^{2}, \frac{\epsilon_{1} \epsilon_{2}}{r}\right]$.

By applying formula (2) to the transformed Breit Hamiltonian, using the generating function $S$ in the form (36), and neglecting the terms of the sixth and higher orders, we obtain

$e^{i S_{e e}} H_{\mathrm{tr}} e^{-i S_{e e}}=H_{\mathrm{tr}}+i\left[S_{e e}, H_{\mathrm{tr}}\right]+\ldots \approx$

$\approx H_{\mathrm{tr}}-\left(\frac{\beta_{1}}{m_{1}^{3}}+\frac{\beta_{2}}{m_{2}^{3}}\right) \frac{\left(\epsilon_{1} \epsilon_{2}\right)^{2}}{8 c^{4} r^{4}}-$

$-\frac{1}{32 m_{1}^{4} c^{4}}\left[\mathbf{p}_{1}^{2},\left[\mathbf{p}_{1}^{2}, \frac{\epsilon_{1} \epsilon_{2}}{r}\right]\right]-\frac{1}{32 m_{2}^{4} c^{4}}\left[\mathbf{p}_{2}^{2},\left[\mathbf{p}_{2}^{2}, \frac{\epsilon_{1} \epsilon_{2}}{r}\right]\right]-$

$-\frac{\beta_{1} \beta_{2}}{32 m_{1} m_{2} c^{4}}\left(\frac{1}{m_{1}}+\frac{1}{m_{2}}\right)\left[\mathbf{p}_{1}^{2},\left[\mathbf{p}_{2}^{2}, \frac{\epsilon_{1} \epsilon_{2}}{r}\right]\right]$.

\footnotetext{
1 Here, we use one common notation $S_{e e}$ for all the even-even generating functions.
}

Note that the term coming after $H_{\text {tr }}$ coincides up to a sign with the one calculated in (22). Thus, transformation (37) removes terms (9a) from the approximate Hamiltonian in the case of the Breit equation. On the other hand, if operator (35) is taken with another factor like $-i 5 / 64 c^{6}$ instead of $-i / 16 c^{6}$, one destroys the last member in (23). But, at the same time, term (22) will be saved out (with another factor).

Let us also consider two further transformations:

$S_{e e}=-\frac{i \beta_{1}}{16 m_{1} m_{2}^{2} c^{6}}\left[[(\mathcal{O E}),(\mathcal{O O})]_{+},(\mathcal{E O})\right]$

$S_{e e}=-\frac{i \beta_{2}}{16 m_{1}^{2} m_{2} c^{6}}\left[[(\mathcal{E O}),(\mathcal{O O})]_{+},(\mathcal{O E})\right]$

Here, the second operator is symmetric to the first one. We apply operator (38b) to modify the transformed Breit Hamiltonian. So, to within terms of the fourth order, we have

$e^{i S_{e e}} H_{\mathrm{tr}} e^{-i S_{e e}}=H_{\mathrm{tr}}+i\left[S_{e e}, H_{\mathrm{tr}}\right]+\ldots \approx$

$\approx H_{\mathrm{tr}}+i\left[S_{e e},(\mathcal{E} \mathcal{E})\right]+i\left[S_{e e}, \frac{\beta_{1}}{2 m_{1} c^{2}}(\mathcal{O} \mathcal{E})^{2}\right]+$

$+i\left[S_{e e}, \frac{\beta_{2}}{2 m_{2} c^{2}}(\mathcal{E O})^{2}\right]$

It is convenient to write out each of the three commutators apart:

$i\left[S_{e e},(\mathcal{E} \mathcal{E})\right]=-\frac{\beta_{2}\left(\epsilon_{1} \epsilon_{2}\right)^{2}}{8 m_{1}^{2} m_{2} c^{4} r^{4}}\left\{2\left[\mathbf{r p}_{1}\right] \boldsymbol{\sigma}_{1}+\left[\mathbf{r p}_{2}\right] \boldsymbol{\sigma}_{1}+\right.$

$\left.+\left(\delta^{i j}-\frac{r^{i} r^{j}}{r^{2}}\right) \sigma_{1}^{i} \sigma_{2}^{j}\right\}$

$i\left[S_{e e}, \frac{\beta_{1}}{2 m_{1} c^{2}}(\mathcal{O} \mathcal{E})^{2}\right]=\frac{\beta_{1} \beta_{2}}{32 m_{1}^{3} m_{2} c^{4}}\left[\boldsymbol{\sigma}_{1}\left(2 i \mathbf{p}_{1}-\nabla\right)\right]^{i} \times$

$\times\left(2 p_{2}^{j}-\left[\boldsymbol{\sigma}_{2} \nabla\right]^{j}\right)\left[\mathbf{p}_{1}^{2},-\frac{\epsilon_{1} \epsilon_{2}}{2 r}\left(\delta^{i j}+\frac{r^{i} r^{j}}{r^{2}}\right)\right]$

$i\left[S_{e e}, \frac{\beta_{2}}{2 m_{2} c^{2}}(\mathcal{E} \mathcal{O})^{2}\right]=\frac{1}{32 m_{1}^{2} m_{2}^{2} c^{4}}\left[\boldsymbol{\sigma}_{1}\left(2 i \mathbf{p}_{1}-\nabla\right)\right]^{i} \times$ 
$\times\left(2 p_{2}^{j}-\left[\boldsymbol{\sigma}_{2} \nabla\right]^{j}\right)\left[\mathbf{p}_{2}^{2},-\frac{\epsilon_{1} \epsilon_{2}}{2 r}\left(\delta^{i j}+\frac{r^{i} r^{j}}{r^{2}}\right)\right]$

One can easily see that commutator (40b) destroys the term which is the second component in the brackets in expression (28). As far as operator (38a) is concerned, it plays the role similar to $(38 \mathrm{~b})$ and leads to the analogous modification of the Hamiltonian but in its symmetric part.

Thus, the higher-order two-body Hamiltonian can be easily modified by unitary "even-even" transformations. With the help of transformations of this kind, one can remove a number of higher-order terms from the Hamiltonian. But, at the same time, it is shown by the example of transformations (35) and (38) that these procedures produce new terms instead of the removed ones (see, e.g., the last term in (37) and (40a, c)).

Obviously, as in the two-particle case, one can modify the transformed one-body Dirac Hamiltonian in a similar way, using an even transformation.

\section{Conclusions}

Thus, on the basis of an extension of the FW method to two-body equations, the expansion of the two-particle Dirac equation to higher orders in $1 / c$ (or in $1 / m$ ) can be easily performed. With the use of the procedure of transformation which is applicable fo the case $m_{1} \neq m_{2}$, we have found the $1 / c^{4}$-order part of the transformed two-body Hamiltonian. We have also shown that the use of the generating functions in the form (6) to transform the original Hamiltonian leads to the appearance of certain extra terms having mass differences in the denominators in $H_{\mathrm{tr}}$, even although both particles are in a positive or negative energy state. These undesirable terms give no contribution to the self-energy of the transformed Hamiltonian and can be canceled by additional unitary transformations with the generating functions in an eveneven form. In addition, transformations of this type simplify $H_{\mathrm{tr}}$ and can be used to modify it in higher orders, in general. The presence of the terms that can be removed by additional transformations (without changing the rest of the terms in $H_{\mathrm{tr}}$ ) is a feature of the expansion of the two-body equations to higher orders.

The treated method gives a straightforward derivation of an effective Hamiltonian of order $\alpha^{6} m c^{2}$ for hydrogen-like atoms. As an example, we have considered the expansion of the Breit equation to order $1 / c^{4}$. The calculated terms form an effective higher- order Hamiltonian for two Coulomb particles having unequal masses. One should note that a number of the terms calculated here in the case of the Breit equation are similar, in many features, to the relativistic corrections in the many-electron higher-order Hamiltonian that has been derived in [14] for an arbitrary light atom.

By expanding a relativistic two-body Hamiltonian, we have in mind, in particular, that it corresponds to the Hamiltonian of the two-particle Dirac equation. However, the obtained results are too far-reaching and can be used for the expansion of relativistic and quasirelativistic two-particle wave equations of other types to higher orders.

1. R.W. Childers, Phys. Rev. D 26, 2902 (1982); F.A.B. Coutinho, W. Glöckle, Y. Nogami, and F.M. Toyama, Can. J. Phys. 66, 769 (1988); W. Królikowski, Acta Phys. Pol. B 20, 119 (1989); W. Glöckle, Y. Nogami, and F.M. Toyama, Prog. Theor. Phys. 81, 706 (1989); A.P. Galeão and P. Leal Ferreira, J. Math. Phys. 33, 2618 (1992); A.G. Nikitin and V.V. Tretynyk, Int. J. Mod. Phys. 12, 4369 (1997); H.W. Crater and P. Van Alstine, Phys. Rev. D 70, 034026 (2004).

2. L.L. Foldy and S.A. Wouthuysen, Phys. Rev. 78, 29 (1950).

3. J.D. Bjorken and S.D. Drell, Relativistic Quantum Mechanics (McGraw-Hill, New York, 1964).

4. C. Itzykson and J.B. Zuber, Quantum Field Theory (McGraw-Hill, New York, 1990).

5. Z.V. Chraplyvy, Phys. Rev. 91, 388 (1953).

6. Z.V. Chraplyvy, Phys. Rev. 92, 1310 (1953).

7. W.A. Barker and F.N. Glover, Phys. Rev. 99, 317 (1955).

8. E. Eriksen, Phys. Rev. 111, 1011 (1958).

9. W. Lucha, F.F. Schöberl, and D. Gromes, Phys. Rep. 200, 127 (1991).

10. Yu.L. Sokolov, Uspekhi Fiz. Nauk 169, 559 (1999); see also P.J. Mohr and B.N. Taylor, Rev. Mod. Phys. 72, 351 (2000).

11. A.P. Martynenko, Yad. Fiz. 71, 126 (2008).

12. A.I. Akhiezer and V.B. Berestetskii, Quantum Electrodynamics (Wiley, New York, 1965).

13. V.B. Berestetskii, E.M. Lifshitz, and L.P. Pitaevskii, Relativistic Quantum Theory (Pergamon Press, Oxford, 1982).

14. K. Pachucki, Phys. Rev. A 71, 012503 (2005).

Received 24.05.10 
РОЗКЛАД ДВОЧАСТИНКОВОГО РІВНЯННЯ ДІРАКА ЗА СТЕПЕНЯМИ 1/с ДО ВИЩИХ ПОРЯДКІВ

\section{O.I. Туровсъкий}

$\mathrm{P}$ е $з$ ю м е

Застосовуючи узагальнення перетворення Фолді-Вутхайзена на двочастинкові хвильові рівняння, розглянуто задачу розкладу двочастинкового гамільтоніана Дірака за степенями $1 /$ c до четвертого порядку включно. Отримано трансформований гамільтоніан у парно-парній формі до порядку $1 / c^{4}$. Показано, що в розкладі у вищих порядках 3'являються особливі члени, які можуть бути виключені додатковими унітарними перетвореннями. Як приклад розглянуто рівняння Брейта для кулонівських частинок та пораховано всі члени порядку $1 / c^{4}$ в його розкладі. Отримані результати можуть бути застосовані для розкладу релятивістських та квазірелятивістських двочастинкових хвильових рівнянь до вищих порядків. 